\section{Oral Presentation Session 1}

\section{STI Surveillance}

\subsection{A TALE OF TWO HALVES; LOW EXTENDED-SPECTRUM CEPHALOSPORIN AND HIGH AZITHROMYCIN RESISTANCE IN NEISSERIA GONORRHOEAE IN EUROPE, 2015}

${ }^{1}$ Michelle Cole, ${ }^{2}$ Gianfranco Spiteri, ${ }^{3}$ Susanne Jacobsson, ${ }^{1}$ Francesco Tripodo, ${ }^{1}$ Neil Woodford, ${ }^{3}$ Magnus Unemo, ${ }^{2}$ Euro-Gasp Network. 'National Infection Service, London, UK; ${ }^{2}$ European Centre of Disease Prevention And Control, Stockholm, Sweden; ${ }^{3}$ Örebro University Hospital, Örebro, Sweden

\subsection{6/sextrans-2017-053264.1}

Introduction The European Gonococcal Antimicrobial Surveillance Programme aims to monitor rates of antimicrobial resistance in Neisseria gonorrhoeae and provide data to inform the European gonorrhoea treatment guidelines; currently ceftriaxone $500 \mathrm{mg}$ plus azithromycin (Az) $2 \mathrm{~g}$ as dual-therapy. This analysis reports on Euro-GASP resistance patterns in 2015 vs. 2014.

Methods For 2015, antimicrobial susceptibility testing (Etest or agar dilution) was performed on 2134 isolates from 24 European countries and interpreted using EUCAST breakpoints. Patient variables associated with resistance were established using univariate logistic regression to estimate odds ratios (ORs).

Results Cefixime resistance (Cef-R) was $1.7 \%$ in 2015 vs. $2.0 \%$ in 2014 . Ceftriaxone resistance was detected in one isolate in 2015, compared with 5 in 2014. The proportion of isolates showing $\mathrm{Az}$ resistance (Az-R) decreased slightly to 7.1\% (7.9\% in 2014). Five isolates displayed high-level Az-R (MIC $\geq 256 \mathrm{mg} / \mathrm{L}$ ) compared with one in 2014. Ciprofloxacin resistance remained high at $49.4 \%(50.7 \%$ in 2014). Cef-R among MSM and females continued to decline to $0.5 \%$ and $1 \%$, respectively, but increased among heterosexual males $(4.1 \%$ vs. $1.7 \%$ in 2014$)$, which was mainly attributable to two countries with high Cef-R (11\%). MSM and heterosexual males had higher levels of Az-R (both 8.1\%) compared with females (4.9\%), but this difference was not significant. Az-R in females increased to $4.9 \%$ from $2.2 \%$ in 2014. An association between previous gonorrhoea infection and Az-R, first observed in 2014 remained in 2015 (OR 2.1, CI 1.2-3.5, $\mathrm{p}<0.01)$

Conclusion The low cephalosporin resistance likely reflects the effectiveness of the current first-line dual-therapy. However, the Az-R is concerning and threatens the effectiveness of this regimen. Furthermore, the increasing cef- $\mathrm{R}$ in heterosexual males needs close monitoring. Increasing AzR and decreasing cephalosporin resistance is occurring globally. Whether the global use of $\mathrm{Az}$ in mono- or dual-therapy is contributing to this picture remains to be elucidated.

\subsection{PREDICTORS OF PERSISTENT AND RECURRENT GENITAL STI SYMPTOMS AT SENTINEL SURVEILLANCE SITES IN SOUTH AFRICA, JANUARY 2015 - JUNE 2016}

${ }^{1}$ Mathebula Rudzani Climentine, ${ }^{1} \mathrm{~L}$ Kuonza, ${ }^{2} \mathrm{~T}$ Kufa-Chakezha. ${ }^{1}$ South Africa Field Epidemiology Training Program, University of Pretoria, Johannesburg, South African Republic, ${ }^{2}$ HIV and STI Centre, National Institute For Communicable Disease, Johannesburg, South African Republic

\subsection{6/sextrans-2017-053264.2}

Introduction South African guidelines recommend a syndromic approach (SA) to manage sexually transmitted infections (STIs). However, SA lacks specificity and may be particularly problematic for patients with persistent or recurrent genital STI symptoms (PRGS) with inability to adequately distinguish the two. The study objectives were to determine the prevalence and risk factors of PRGS.

Methods We conducted a cross-sectional study at 7 STI sentinel sites in 5 provinces. Demographic, clinical information and clinical specimens were collected. Multivariable logistic regression analysis was used. PRGS was defined as patients who self-reported non-resolution of genital symptoms in the preceding three months or previous treatment for the same genital STI symptoms in the preceding 12 months.

Results Of 1397 eligible patients, 28.9\% reported PRGS. Among those with PRGS, recurrence was more common $90.1 \%$ than persistence $21.8 \%$ with $13.2 \%$ reporting both. The median age of those who reported PRGS was 28 years [IQR: 24-33], with 56.6\% females. Among those with PRGS, $49.3 \%$ had vaginal discharge syndrome (VDS), 33.4\% had male urethral syndrome (MUS) and 17.1\% had genital ulcer syndrome (GUS). The most common pathogens identified among VDS, MUS and GUS patients with PRGS were bacterial vaginosis (60\%), Neisseria gonorrhoea (81.5\%), and herpes simplex virus 2 (57.5\%), respectively. There were no pathogens detected in $19.8 \%$ patients. In multivariable analysis, being HIV-positive (adjusted odds ratio $[\mathrm{aOR}]=1.52$, 95\% CI 1.16-2.00), having VDS $(\mathrm{aOR}=1.66,95 \%$ CI $1.23-2.25)$, being seen at a study site in Gauteng province $(\mathrm{aOR}=0.63$, 95\% CI $0.45-0.89)$ and age between 25-34 years $(\mathrm{aOR}=1.43$, 95\% CI 1.06-1.92) were significantly associated with having PRGS, after adjusting for potential covariates.

Conclusion We found high prevalence of PRGS, associated with HIV infection and VDS among young adults. We therefore recommend better integration of HIV and STI management, increasing HIV testing, and review of SA for VDS to include microbiological testing for pathogens associated with PRGS.

\subsection{ABSTRACT WITHDRAWN}

001.4 HIGH PREVALENCE OF HEPATITIS C VIRUS AMONG HIV NEGATIVE MSM IN AMSTERDAM PREP PROJECT

${ }^{1}$ Roel Achterbergh, 'Elske Hoornenborg, 'Maarten F Schim Van Der Loeff, ' ${ }^{1}$ di Davidovich, ${ }^{1}$ Arjan Hogewoning, ${ }^{2}$ Henry Jc De Vries, ${ }^{1}$ Maria Prins, ${ }^{2}$ Janke Schinkel, ${ }^{3}$ Thijs Jw Van De Laar. 'Public Health Serice Amsterdam, Amsterdam, The Netherlands; ${ }^{2}$ Amsterdam Medical Centre, Amsterdam, The Netherlands; ${ }^{3}$ Sanquin Blood Supply, Amsterdam, The Netherlands

10.1136/sextrans-2017-053264.3 
Introduction Since 2000, hepatitis C virus (HCV) has emerged as a sexually transmitted infection among men who have sex with men (MSM). Although the reported HCV epidemic has largely been confined to HIV infected MSM, spread to HIV negative MSM might have gone unnoticed.

Methods HIV negative MSM at high risk for acquiring HIV who enrolled in the Amsterdam Pre-Exposure Prophylaxis (AMPrEP) demonstration project at the Public Health Service of Amsterdam were tested for the presence of HCV antibodies and HCV RNA. If positive for HCV RNA, part of the HCV NS5B gene (709 bp) was sequenced. Maximum likelihood phylogenies (GTR substitution model) were constructed to compare HCV sequences from HIV negative AMPrEP participants, Dutch HIV positive MSM with acute or chronic HCV infection ( $n=246$; period 2000-2015) and Dutch risk groups other than MSM ( $n=153$; period 2000-2015). Bootstrap values $>70 \%$ define robust phylogenetic clusters.

Results By June 2016, all 376 HIV negative MSM had been enrolled in AMPrEP; 18 (4.8\%, 95\% CI 2.8\%-7.5\%) were positive for anti-HCV or HCV-RNA at baseline. Of those, 15/ 18 (83\%) had detectable HCV-RNA, including one without detectable anti-HCV. HCV genotyping showed genotype 1a (73\%), 4d (20\%) and 2b (7\%). Of the 15 participants with HCV RNA, 13 (87\%) were part of 6 robust MSM-specific HCV clades containing MSM with and without HIV. This included 9/11 HIV negative MSM infected with HCV-1a (Figure 1), and all 4 MSM infected with HCV-4d and HCV-2b. Four out of $17(24 \%) \mathrm{HCV}$ positive participants reported injecting drugs in the 3 months preceding PrEP start, compared to $11 / 354(3.1 \%)$ among HCV negative participants.

Conclusion The HCV prevalence of $4.8 \%$ among HIV-negative MSM eligible for PrEP was higher than the prevalence around $1 \%$ previously observed among Dutch HIV negative MSM attending an STI clinic and not on PrEP. HCV-mono-infected MSM were infected with the same MSM-specific HCV strains circulating among HCV/HIV co-infected MSM, suggesting spread from HIV positive to high-risk HIV negative MSM. Routine HCV testing should be offered to MSM at high risk for HIV and included in PrEP guidelines.

\subsection{ORIGIN AND PREDICTORS OF EARLY REPEAT INFECTIONS AMONG HIV NEGATIVE WOMEN WITH TRICHOMONAS VAGINALIS RECEIVING A 2 G DOSE OF METRONIDAZOLE}

${ }^{1}$ Patricia Kissinger, ${ }^{2}$ Christina Muzny, ${ }^{3}$ Leandro Mena, ${ }^{4}$ Rebecca Lillis, ${ }^{2}$ Jane Schwebke, ${ }^{3}$ Laura Beauchamps, ${ }^{4}$ Stephanie N Taylor, ${ }^{1}$ Norine Schmidt, 'Lauren Ostrenga, ${ }^{5} \mathrm{~W}$ Evan Secor, 'David H Martin. 'Tulane University School of Public Health And Tropical Medicine, Department of Epidemiology, New Orleans, USA; ${ }^{2}$ University of Alabama At Birmingham, Division of Infectious Diseases, Birmingham, USA; ${ }^{3}$ Univeristy of Mississippi Medical Centre, Department of Medicine, Jackson, USA; ${ }^{4}$ Louisiana State University Health Sciences Centre, Section of Infectious Diseases, New Orleans, USA; ${ }^{5}$ Centres for Disease Control and Prevention, Division of Parasitic Diseases And Malaria, Atlanta, USA

\subsection{6/sextrans-2017-053264.4}

Introduction A recent meta-analysis demonstrated superiority of multi-dose metronidazole (MTZ) over the CDC and WHO recommended $2 \mathrm{~g}$ dose for the treatment of T. vaginalis (TV). Another study among HIV+ women with TV found higher test-of-cure (TOC)+ rates among women who had asymptomatic bacterial vaginosis (BV) than those without BV. The purpose of this study was to measure the TOC $\mathrm{TV}+$ rate and to examine if the presence of $\mathrm{BV}$ influenced that rate.
Methods HIV-/TV+ women treated with $2 \mathrm{~g}$ oral directly observed MTZ and who completed their TOC visit 3-12 weeks post treatment were included. Women were tested for TV using NAAT and surveyed via computer at baseline and TOC. Nugent scores $\geq 7$, calculated from vaginal gram stain, were considered $\mathrm{BV}+$. MTZ susceptibility testing was performed on TOC TV+ specimens.

Results Of $227 \mathrm{TV}+$ women included baseline the mean age was 31.3 (S.D. 9.9), 95.2\% were African American, 39.3\% had multiple male partners in the prior 3 months, $32.3 \%$ regularly smoked, $19.4 \%$ were binge drinkers, $48.9 \%$ had BV and $5.4 \%$ had yeast on the gram stain. At TOC, $19.8 \%$ were NAAT TV + . Of the 45 TOC-TV+ women, 44 provided sexual exposure information and 10/44 (24.4\%) reported sexual re-exposure to baseline partner or sexual exposure to a new partner. Two of $26(7.7 \%)$ TOC+ specimens that underwent susceptibility testing had low to moderate MTZ resistance $(50-100 \mathrm{ug} / \mathrm{ml})$. There were no differences in TOC NAAT+ rates by $\mathrm{BV}$, sexual re-exposure to a baseline partner, sexual exposure to a new partner, regular smoking, binge drinking, or by the presence of yeast $(p>0.22)$.

Conclusion TOC NAAT + rate after 2 g MTZ dose was high $(19.8 \%)$ and isolates from these women were susceptible to MTZ (94.3\%). Most TOC+ women (75.6\%) reported no sexual exposure/re-exposure during follow-up suggesting that most cases were treatment failures. Selected behavioural factors and BV did not did not appear to influence TV treatment. The $2 \mathrm{~g}$ MTZ dose for TV recommended by CDC and WHO, should be reevaluated in light of more sensitive NAAT.

\subsection{EVALUATING CHLAMYDIA TRENDS IN THE UNITED STATES 2000-2015 USING A PAIR FORMATION TRANSMISSION MODEL}

${ }^{1}$ Minttu M Rönn, ${ }^{1}$ Ashleigh Tuite, ${ }^{1}$ Nicolas A Menzies, ${ }^{2}$ Thomas Gift, ${ }^{2}$ Harrell Chesson, ${ }^{2}$ Elizabeth Torrone, ${ }^{1}$ Emory E Wolf, ${ }^{1}$ Kara Galer, ${ }^{3}$ Katherine Hsu, ${ }^{1}$ Joshua A Salomon. ${ }^{1}$ Harvard T. H. Chan School of Public Health, Boston, USA; ${ }^{2}$ Centres for Disease Control and Prevention, Atlanta, USA; ${ }^{3}$ Massachusetts Department of Public Health, Boston, USA

\subsection{6/sextrans-2017-053264.5}

Introduction In the United States reported cases of chlamydia have increased since reporting began, due in part to increased screening. However, the implication of these trends for the population prevalence remains unclear. We aimed to understand and reconcile the epidemiological trends, and examine counterfactuals.

Methods We developed a deterministic heterosexual pair formation model to simulate chlamydia epidemiology in the US heterosexual population aged $15-54 \mathrm{y}$. The pair formation model accounts explicitly for sexual partnership dynamics, such as re-infection within the partnership, and the model is stratified by age, risk and relationship type (long-term v. casual). We used a Bayesian approach to calibrate model parameters (including time-varying screening, reporting and test sensitivity) to age- and sex-specific national case report rates from 2000-2015 (ages 15-54y), lab-measured population prevalence estimates from NHANES 1999-2014 (15-39y), and sexual behaviour data from the Youth Risk Behaviour Survey (15$18 \mathrm{y})$.

Results Model estimates were able to reproduce both chlamydia prevalence and reported case rates. Results indicate an increase in chlamydia screening in women. 